\title{
Polymorphisms of STAT4 and the risk of inflammatory bowel disease: A case-control study in Chinese Han population
}

\author{
HOUBAO ZHU ${ }^{1 *}$, JIANBING LIU $^{1 *}$, HONGXIN ZHANG $^{2}$, ZHENGTING WANG $^{3}$, \\ JIE LIU ${ }^{4}$, SHUNYUAN LU ${ }^{2}$, WANGYANG XU' ${ }^{1}$, JIE ZHONG ${ }^{3}$ and ZHUGANG WANG ${ }^{1,2,5}$ \\ ${ }^{1}$ Department of Medical Genetics, E-Institutes of Shanghai Universities, Shanghai Jiao Tong University \\ School of Medicine; ${ }^{2}$ Research Center for Experimental Medicine, State Key Laboratory of Medical Genomics, \\ Shanghai Institute of Hematology; ${ }^{3}$ Department of Gastroenterology; ${ }^{4}$ Shanghai Key Laboratory for Prevention \\ and Treatment of Bone and Joint Diseases with Integrated Chinese-Western Medicine, Shanghai Institute of Orthopaedics \\ and Traumatology, Department of Orthopaedics, Rui-Jin Hospital Affiliated to Shanghai Jiao Tong University \\ School of Medicine; ${ }^{5}$ Shanghai Research Center for Model Organisms, Shanghai, P.R. China
}

Received October 14, 2012; Accepted January 3, 2013

DOI: $10.3892 /$ br.2013.59

\begin{abstract}
Signal transducer and activator of transcription 4 (STAT4) is a transcription factor involved in the signaling pathways of several cytokines, playing an essential role in the development of inflammation in various immune-mediated diseases. Genetic association studies have shown that the STAT4 gene was significantly associated with inflammatory bowel disease (IBD) in Spanish and Caucasian populations. However, these associations in other ethnic populations remain unknown. In the present study, we evaluated the role of the STAT4 rs7574865 and rs7582694 polymorphisms on IBD in 562 unrelated Chinese Han subjects by assessing distributions of genotypes and allele frequencies. Results showed that neither rs7574865 [Crohn's disease (CD): $\mathrm{P}=0.66$, odds ratio $(\mathrm{OR})=0.95,95 \%$ confidence interval (CI) 0.74-1.21; ulcerative colitis (UC): $\mathrm{P}=0.43, \mathrm{OR}=0.85$, 95\% CI 0.56-1.28; IBD: $\mathrm{P}=0.52, \mathrm{OR}=0.93,95 \%$ CI $0.73-1.17]$ nor rs7582694 (CD: $\mathrm{P}=0.40, \mathrm{OR}=1.12,95 \% \mathrm{CI} 0.86-1.44$; UC: $\mathrm{P}=0.50, \mathrm{OR}=0.86,95 \%$ CI 0.56-1.33; IBD: $\mathrm{P}=0.62, \mathrm{OR}=1.06$, 95\% CI 0.83-1.36) was significantly associated with IBD, although the genotype frequency of rs7574865 varied in patients and the controls. In conclusion, our data did not
\end{abstract}

Correspondence to: Professor Zhugang Wang, Department of Medical Genetics, E-Institutes of Shanghai Universities, Shanghai Jiao Tong University School of Medicine, 280 South Chongqing Road, Shanghai 200025, P.R. China

E-mail: 0117109043@sjtu.edu.cn

${ }^{*}$ Contributed equally

Key words: inflammatory bowel disease, Chinese Han population, single-nucleotide polymorphisms, signal transducer and activator of transcription 4 support that STAT4 variants contribute to IBD susceptibility in the Chinese Han population.

\section{Introduction}

Inflammatory bowel disease (IBD) is a series of inflammatory conditions of the colon and small intestine comprising Crohn's disease (CD) and ulcerative colitis (UC) (1-4). IBD, with a complex pathogenesis influenced by a combination of genetic risk factors and environmental events, is highly heritable, thus investigations into the genetic susceptibility of this disease are ongoing $(5,6)$. Numerous loci, such as NOD2, IL23R, ATG16L1 and IRGM, are associated with IBD susceptibility (7-14). However, the genetic polymorphisms located within these genes are not sufficient to adequately explain the pathogenesis and development of IBD and its various phenotypes.

Signal transducer and activator of transcription 4 (STAT4), a transcription factor whose gene is located on chromosome $2 q 33$, is important in the development of inflammation of various immune-mediated diseases (15). STAT4 variation has been reported to be significantly associated with rheumatoid arthritis (RA) and systemic lupus erythematosus (SLE) (16). The association of STAT4 haplotype characterized by the rs7574865 polymorphism with IBD was originally reported by Martinez et al (17) in a Spanish population, a study that was replicated by Diaz-Gallo et al (18). Glas et al (19) reported that rs7574865 polymorphism was associated with colonic CD and early disease onset in Caucasians.

These associations need to be confirmed by replication studies, particularly in other ethnic populations. Additionally, the differences in risk allele frequencies and linkage disequilibrium structure in ethnicities may provide insights to refine the association signal and identify the true risk variant. Therefore, the aim of the present study was to evaluate whether the previously identified STAT4 single-nucleotide polymorphisms (SNPs) are associated with IBD susceptibility in a population-based Chinese Han cohort including 562 unrelated individuals (232 CD, 56 UC and 274 healthy controls). 


\section{Patients and methods}

Human subjects. A total of 288 unrelated Chinese patients with IBD (232 with CD and 56 with UC) and 274 matched healthy controls were included in this study. The patients were recruited from the Department of Gastroenterology of the Ruijin Hospital Affiliated to the Shanghai Jiaotong University School of Medicine. The patients were diagnosed by senior physicians according to standard clinical, endoscopic, radiologic and histological criteria $(20,21)$. Healthy individuals with no history of digestive system disease, unrelated to each other or to the patients were randomly selected under routine health screening as controls (Table I). The study was approved by the Research Ethics Committee of the Ruijin Hospital and the enrolled patients provided informed consent to participate in the study.

STAT4 genotyping. Genomic DNA was extracted from whole blood samples using QIAamp blood extraction kit (Qiagen, Hilden, Germany), following the manufacturer's instructions. The DNA samples were genotyped for the rs7574865 and rs7582694 polymorphisms via polymerase chain reaction (PCR) with sequence-specific primers. The primer sequences, designed using the genomic sequences in the GenBank (http:// www.ncbi.nlm.nih.gov) are listed in Table II. Genotyping of the amplified products was assessed for the presence/absence of PCR amplicons specific to the particular alleles by using a standard $2 \%$ agarose gel electrophoresis with ethidium bromide staining. Then, $\sim 10 \%$ samples were confirmed using Sanger sequencing.

Statistical analysis. Hardy-Weinberg equilibrium testing (HWE), P-value computations ( $\mathrm{P}>0.05)$, in the healthy control and patient groups, calculations of allelic and genotypic associations of SNPs with susceptibility to IBD were performed using the SHEsis software (http://analysis.bio-X.cn) $(22,23)$. The tests were two-tailed and $\mathrm{P}<0.05$ was considered to indicate a statistically significant difference.

\section{Results}

Patients. To evaluate the association of the STAT4 polymorphisms with IBD in the Chinese Han population, we
Table I. Characteristics of the study samples.

\begin{tabular}{lccc}
\hline & \multicolumn{2}{c}{ Patients } & \\
\cline { 2 - 4 } Characteristics & UC & CD & Control subjects \\
\hline Number & 56 & 232 & 274 \\
Age (years) & & & \\
Mean \pm SD & $42.5 \pm 16.7$ & $33.6 \pm 13.5$ & $62.1 \pm 10.1$ \\
Range & $3-77$ & $1-76$ & $37-88$ \\
Male/female & $33 / 23$ & $149 / 83$ & $119 / 155$ \\
\hline
\end{tabular}

UC, ulcerative colitis; CD, Crohn's disease; SD, standard deviation.

genotyped SNP rs7574865 and rs7582694 among 232 CD and 56 UC patients, as well as 274 healthy controls. Characteristics of the study subjects are shown in Table I.

Genotypic and allele frequencies. Distributions of the genotype and allele frequencies of the two SNPs between the cases and healthy controls are shown in Table III. Results revealed that the STAT4 rs7574865 genotype was significantly different between $\mathrm{CD} / \mathrm{IBD}$ patients and the controls. However, the association analysis showed that neither of the two SNPs was significantly associated with IBD susceptibility (rs7574865 $\mathrm{CD}: \mathrm{P}=0.66, \mathrm{OR}=0.95,95 \%$ CI $0.74-1.21$; $\mathrm{UC}: \mathrm{P}=0.43$, $\mathrm{OR}=0.85,95 \%$ CI $0.56-1.28$; IBD: $\mathrm{P}=0.52, \mathrm{OR}=0.93,95 \% \mathrm{CI}$ 0.73-1.17; rs7582694 CD: $\mathrm{P}=0.40, \mathrm{OR}=1.12,95 \%$ CI 0.86-1.44; UC: $\mathrm{P}=0.50, \mathrm{OR}=0.86,95 \%$ CI $0.56-1.33$; IBD: $\mathrm{P}=0.62$, $\mathrm{OR}=1.06,95 \%$ CI 0.83-1.36) (Table III).

\section{Discussion}

IBD is a series of inflammatory conditions of the colon and small intestine, characterized by a combination of genetic risk factors and environmental events. Numerous gene variants are reportedly associated with IBD or IBD-related phenotypes (7-14). However, these variants are not sufficient to adequately explain the pathogenesis and development of IBD and its various phenotypes. The STAT4 gene has been previously reported to be significantly associated with certain autoim-

Table II. Primer sequences used for STAT4 genotyping.

\begin{tabular}{lll}
\hline Polymorphism & \multicolumn{1}{c}{ Description } & \multicolumn{1}{c}{ Primer sequences } \\
\hline rs7574865 & Internal control forward primer & CTGTTAATACGGATGTCT \\
& Common reverse primer & ACTTCTTGCTTTAGGAGT \\
& Specific primer G & AAGTTGGTGACCAAAATGTG \\
rs7582694 & Specific primer T & AAGTTGGTGACCAAAATGTT \\
& Internal control forward primer & TGGAATCCAACTCTTCTCAGCC \\
& Common reverse primer & AAAATGTTACCAATGCTTATCT \\
& Specific primer C & TTCATGAAGGGATGACACATAC \\
& Specific primer G & TTCATGAAGGGATGACACATAG
\end{tabular}

STAT4, signal transducer and activator of transcription 4. 


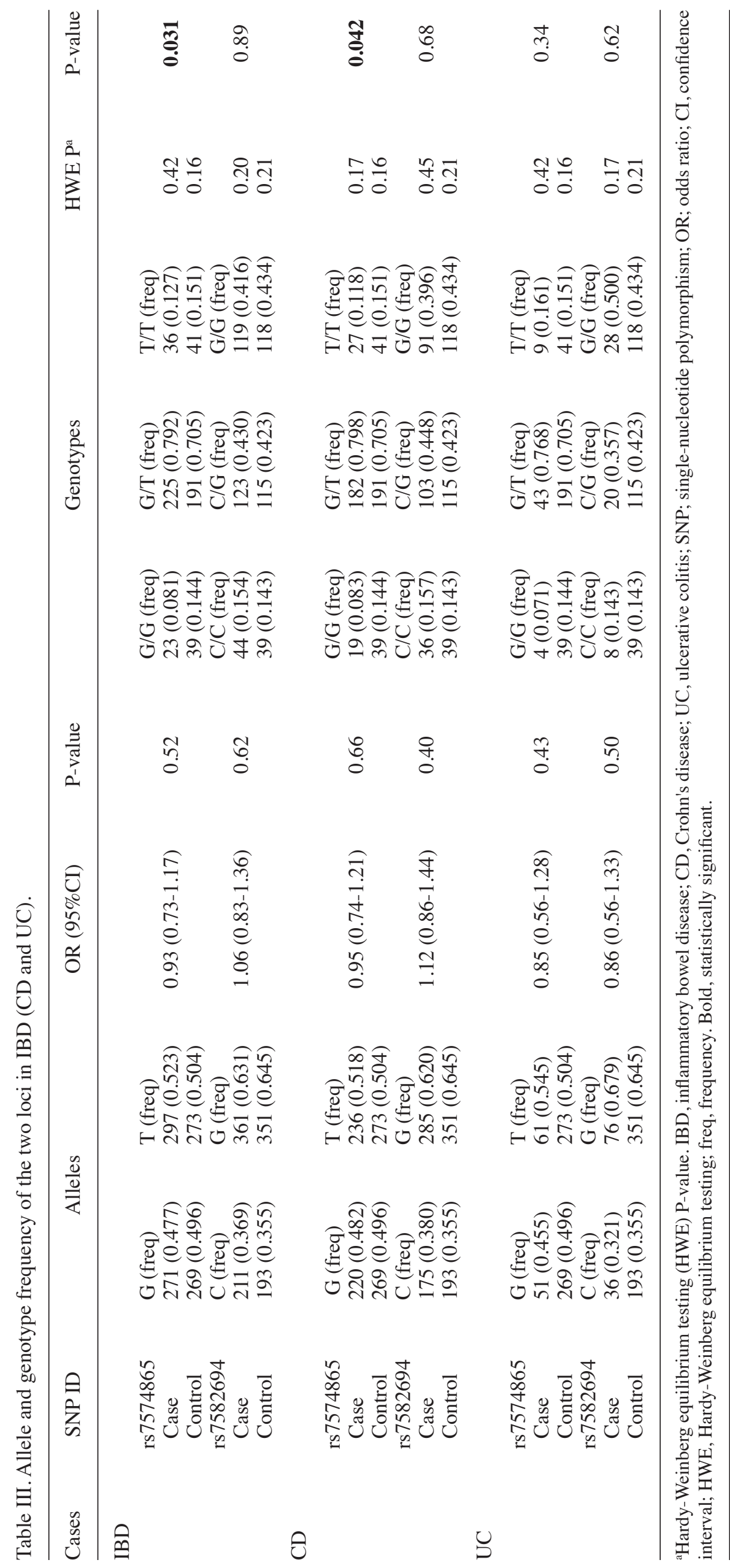


mune diseases such as SLE, RA $(16,24,25)$ and psoriasis $(24,26)$.

The Chinese Han is one of the largest populations in the world. Evaluation of the association of risk factors with specific diseases is crucial. In this study, we present a genotype-phenotype analysis of STAT4 gene polymorphisms in an IBD cohort. This study did not reproduce the association of STAT4 variants with susceptibility to IBD in the Chinese population. However, we found the genotype frequency of rs7574865 to vary significantly in $\mathrm{CD} / \mathrm{IBD}$ patients and the healthy controls. Previous studies have shown that the STAT4 SNP rs7574865 was significantly associated with RA (16), IBD and type I diabetes (17). Additionally, the association analysis of rs7574865 with IBD was carried out independently by several groups based on various ethnic populations. Unlike the original findings of Martinez et al (17), Glas et al (19), who analyzed 2,704 individuals of Caucasian origin, hypothesized that rs7574865 was associated with CD and early disease onset, but not with UC. Nevertheless, in another independent Spanish cohort Diaz-Gallo et al (18) observed that rs7574865 was associated with UC but not CD. A further discrepancy was noted by Moon et al (27) who analyzed eight STAT4 SNPs in 657 unrelated Korean participants, and did not detect any statistically significant association between rs7574865 and $\mathrm{CD}$ or UC. These varying results may be due to the variation of complex circumstances and extended genetic background.

Other SNPs of STAT4, such as rs7582694, also analyzed in our study, may affect several autoimmune diseases in various ethic populations (28-30). However, rs7582694 was not found to be associated with IBD. Although numerous gene variants are reportedly associated with IBD susceptibility (7,10-14), a re-evaluation of the association is required in additional ethic populations. Lack of replication has long been a challenge in genetic association studies of IBD.

Taken together, although our study was sufficiently powered, there was no evidence to show that the previously reported common variants rs7574865 and rs7582694 in the STAT4 gene increased the risk of IBD in a Chinese Han population. However, this study has a limitation. Although the two SNPs were not associated with IBD susceptibility and phenotypes, the relatively small study population caused the weak diversity of the genotype frequency of rs7574865 in the CD patients and controls. The association analysis in our study should be replicated in a larger sample-sized study of Chinese patients, as well as in other ethnic populations.

\section{Acknowledgements}

This study was funded by the Science and Technology Commission of the Municipality of Shanghai (grant no. 10JC1410300 to Z.G. Wang) and the National Natural Science Foundation of China (grant no. 31000408 to H.X. Zhang and 81201365 to W.Y. Xu). The authors would like to thank the members of the cohort for their devoted contribution to a scientific discovery.

\section{References}

1. Baumgart DC and Carding SR: Inflammatory bowel disease: cause and immunobiology. Lancet 369: 1627-1640, 2007.
2. Baumgart DC and Sandborn WJ: Inflammatory bowel disease: clinical aspects and established and evolving therapies. Lancet 369: 1641-1657, 2007.

3. Podolsky DK: Inflammatory bowel disease. N Engl J Med 347: 417-429, 2002

4. Xavier RJ and Podolsky DK: Unravelling the pathogenesis of inflammatory bowel disease. Nature 448: 427-434, 2007.

5. Cho JH: The genetics and immunopathogenesis of inflammatory bowel disease. Nat Rev Immunol 8: 458-466, 2008.

6. Van Limbergen J, Wilson DC and Satsangi J: The genetics of Crohn's disease. Annu Rev Genomics Hum Genet 10: 89-116, 2009.

7. Duerr RH, Taylor KD, Brant SR, et al: A genome-wide association study identifies IL23R as an inflammatory bowel disease gene. Science 314: 1461-1463, 2006.

8. Glas J, Konrad A, Schmechel S, et al: The ATG16L1 gene variants rs2241879 and rs2241880 (T300A) are strongly associated with susceptibility to Crohn's disease in the German population. Am J Gastroenterol 103: 682-691, 2008.

9. Glas J, Seiderer J, Wetzke M, et al: rs1004819 is the main disease-associated IL23R variant in German Crohn's disease patients: combined analysis of IL23R, CARD15, and OCTN1/2 variants. PLoS One 2: e819, 2007.

10. Hampe J, Franke A, Rosenstiel P, et al: A genome-wide association scan of nonsynonymous SNPs identifies a susceptibility variant for Crohn disease in ATG16L1. Nat Genet 39: 207-211, 2007.

11. Hugot JP, Chamaillard M, Zouali H, et al: Association of NOD2 leucine-rich repeat variants with susceptibility to Crohn's disease. Nature 411: 599-603, 2001.

12. Ogura Y, Bonen DK, Inohara N, et al: A frameshift mutation in NOD2 associated with susceptibility to Crohn's disease. Nature 411: 603-606, 2001.

13. Parkes M, Barrett JC, Prescott NJ, et al: Sequence variants in the autophagy gene IRGM and multiple other replicating loci contribute to Crohn's disease susceptibility. Nat Genet 39: 830-832, 2007.

14. Rioux JD, Xavier RJ, Taylor KD, et al: Genome-wide association study identifies new susceptibility loci for Crohn disease and implicates autophagy in disease pathogenesis. Nat Genet 39: 596-604, 2007.

15. Ihle JN: The Stat family in cytokine signaling. Curr Opin Cell Biol 13: 211-217, 2001

16. Remmers EF, Plenge RM, Lee AT, et al: STAT4 and the risk of rheumatoid arthritis and systemic lupus erythematosus. N Engl J Med 357: 977-986, 2007.

17. Martinez A, Varade J, Marquez A, et al: Association of the STAT4 gene with increased susceptibility for some immune-mediated diseases. Arthritis Rheum 58: 2598-2602, 2008.

18. Diaz-Gallo LM, Palomino-Morales RJ, Gomez-Garcia M, et al: STAT4 gene influences genetic predisposition to ulcerative colitis but not Crohn's disease in the Spanish population: a replication study. Hum Immunol 71: 515-519, 2010.

19. Glas J, Seiderer J, Nagy M, et al: Evidence for STAT4 as a common autoimmune gene: rs7574865 is associated with colonic Crohn's disease and early disease onset. PLoS One 5: e10373, 2010.

20. Satsangi J, Silverberg MS, Vermeire S and Colombel JF: The Montreal classification of inflammatory bowel disease: controversies, consensus, and implications. Gut 55: 749-753, 2006.

21. Silverberg MS, Satsangi J, Ahmad T, et al: Toward an integrated clinical, molecular and serological classification of inflammatory bowel disease: report of a Working Party of the 2005 Montreal World Congress of Gastroenterology. Can J Gastroenterol 19 (Suppl A): S5-S36, 2005.

22. Li Z, Zhang Z, He Z, et al: A partition-ligation-combination-subdivision $\mathrm{EM}$ algorithm for haplotype inference with multiallelic markers: update of the SHEsis (http://analysis.bio-x. cn). Cell Res 19: 519-523, 2009.

23. Shi YY and He L: SHEsis, a powerful software platform for analyses of linkage disequilibrium, haplotype construction, and genetic association at polymorphism loci. Cell Res 15: 97-98, 2005.

24. Korman BD, Kastner DL, Gregersen PK and Remmers EF: STAT4: genetics, mechanisms, and implications for autoimmunity. Curr Allergy Asthma Rep 8: 398-403, 2008.

25. Orozco G, Alizadeh BZ, Delgado-Vega AM, et al: Association of STAT4 with rheumatoid arthritis: a replication study in three European populations. Arthritis Rheum 58: 1974-1980, 2008. 
26. Zervou MI, Goulielmos GN, Castro-Giner F, Tosca AD and Krueger-Krasagakis S: STAT4 gene polymorphism is associated with psoriasis in the genetically homogeneous population of Crete, Greece. Hum Immunol 70: 738-741, 2009

27. Moon CM, Cheon JH, Kim SW, et al: Association of signal transducer and activator of transcription 4 genetic variants with extra-intestinal manifestations in inflammatory bowel disease. Life Sci 86: 661-667, 2010.

28. Hellquist A, Sandling JK, Zucchelli M, et al: Variation in STAT4 is associated with systemic lupus erythematosus in a Finnish family cohort. Ann Rheum Dis 69: 883-886, 2010.
29. Luan H, Li P, Cao C, et al: A single-nucleotide polymorphism of the STAT4 gene is associated with systemic lupus erythematosus (SLE) in female Chinese population. Rheumatol Int 32: 1251-1255, 2012.

30. Sigurdsson S, Nordmark G, Garnier S, et al: A risk haplotype of STAT4 for systemic lupus erythematosus is over-expressed, correlates with anti-dsDNA and shows additive effects with two risk alleles of IRF5. Hum Mol Genet 17: 2868-2876, 2008. 